Draft version September 23, 2021

Preprint typeset using $\mathrm{IAT}_{\mathrm{E}} \mathrm{X}$ style emulateapj v. 5/2/11

\title{
AN INCONSISTENCY IN STANDARD MAXIMUM LIKELIHOOD ESTIMATION OF BULK FLOWS
}

\author{
Adi Nusser \\ Physics Department and the Asher Space Science Institute-Technion, Haifa 32000, Israel \\ e-mail: adi@physics.technion.ac.il \\ Draft version September 23, 2021
}

\begin{abstract}
Maximum Likelihood estimation of the bulk flow from radial peculiar motions of galaxies, generally assumes a constant velocity field inside the survey volume. The assumption is inconsistent with the definition of the bulk flow as the average of the peculiar velocity field over the relevant volume. This follows from a straightforward mathematical relation between the bulk flow of a sphere and the velocity potential on its surface. The inconsistency exists also for ideal data with exact radial velocities and full spatial coverage. Based on the same relation we propose a simple modification to correct for this inconsistency.
\end{abstract}

Subject headings: Cosmology: theory, observations, large scale structure of the universe, dark matter

\section{INTRODUCTION}

In the standard cosmological paradigm, galaxies share the same peculiar velocity field (deviations from a pure Hubble flow), $\boldsymbol{v}$, as the underlying dark matter, at least on large scales away from virialized regions. Thus, although the distribution of galaxies may not be an honest tracer of the underlying density field, their motions offer, in principle, an unbiased probe of the gravitationally dominant dark matter. The bulk flow, defined as the average peculiar velocity in a volume of space, is one of the common statistical measures of the velocity field. Usually, the bulk flow of a sphere of comoving radius $r$ centered at the observer is considered,

$$
\mathbf{B}(r)=\frac{3}{4 \pi r^{3}} \int_{0}^{r} \boldsymbol{v}\left(\boldsymbol{r}^{\prime}\right) \mathrm{d}^{3} r^{\prime} .
$$

The is the most basic velocity moment beyond the trivial monopole term describing a purely radial flow. Nonetheless, its estimation from observational data is a nontrivial matter. The relevant observations are very challenging and provide only the radial (line of sight component) peculiar motions of a relatively small number (a few $\sim 10^{3}$ ) of galaxies, within $\lesssim 200 h^{-1}$ Mpc (e.g Mas-

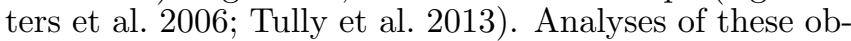
servations could easily be plagued by systematic biases due to sparseness of data and varying quality of distance measurements (Lynden-Bell et al. 1988). The bulk flow is essentially a large scale moment and hence it is particularly prone for systematics which may masquerade as a real signal. Putting these potential biases aside, we focus here on a single point related to estimating $\mathbf{B}$ from velocity data by means of a Maximum Likelihood (ML) estimation (e.g. Kaiser 1988). We address the issue in view of a very simple relation between $\mathbf{B}(r)$ and the velocity potential on the surface of the sphere of radius $r$. Assuming a constant bulk flow in the sphere, the ML estimation does not yield the average flow as given in (1). However, we will see that there exists a simple remedy for this inconsistency.

\section{BASICS}

Let $\hat{\boldsymbol{x}}, \hat{\boldsymbol{y}}$ and $\hat{\boldsymbol{z}}$ be unit vectors in the three axes of fixed Cartesian system. The radial direction is indicated by the unit vector $\hat{\boldsymbol{r}}$ and the projections of $\hat{\boldsymbol{r}}$ into the cartesian axes are $\hat{n}^{\alpha}$, where $\alpha$ runs over $x, y$ and $z$, corresponding to $\hat{n}^{x}=\hat{\boldsymbol{r}} \cdot \hat{\boldsymbol{x}}=\sin \theta \cos \varphi, \hat{n}^{y}=\hat{\boldsymbol{r}} \cdot \hat{\boldsymbol{y}}=\sin \theta \sin \varphi$ and $\hat{n}^{z}=\hat{\boldsymbol{r}} \cdot \hat{\boldsymbol{z}}=\cos \theta$. These projections can be represented as combinations of the $l=1$ spherical harmonics, $\hat{n}_{z}=\sqrt{4 \pi / 3} Y_{10}, \hat{n}_{y}=i \sqrt{2 \pi / 3}\left(Y_{11}+Y_{1-1}\right)$ and $\hat{n}_{x}=-\sqrt{2 \pi / 3}\left(Y_{11}-Y_{1-1}\right)$.

We assume a potential flow, i.e. the peculiar velocity can be written as $\boldsymbol{v}(\boldsymbol{r})=-\boldsymbol{\nabla} \phi(\boldsymbol{r})$, where $\phi$ is the velocity potential function. If we expand the angular dependence of $\phi$ into spherical harmonics, $Y_{l m}$, then it is easy to see that the only term contributing to $\mathbf{B}$ in $(\sqrt{6})$ is the dipole, $l=1$. The expansion by means of $l=1$ spherical harmonics is entirely equivalent to a representation in terms of the angular functions $n^{\alpha}(\theta, \varphi)$

$$
\phi(r, \hat{\boldsymbol{r}})=\sum_{\alpha \in x, y, z}^{3} \phi^{\alpha}(r) \hat{n}^{\alpha},
$$

where

$$
\phi^{\alpha}(r)=\frac{3}{4 \pi} \int \mathrm{d} \Omega \phi(\boldsymbol{r}) n^{\alpha}
$$

thanks to orthogonality conditions $\int \mathrm{d} \Omega n^{\alpha} n^{\beta}=$ $4 \pi / 3 \delta_{\alpha \beta}^{\mathrm{K}}$. The corresponding representation of the peculiar velocity field is

$$
\boldsymbol{v}=-\sum_{\alpha}\left[\frac{\mathrm{d} \phi^{\alpha}}{\mathrm{d} r} \hat{n}^{\alpha} \hat{\boldsymbol{r}}+\frac{\phi^{\alpha}}{r} \nabla_{\theta, \varphi} \hat{n}^{\alpha}\right],
$$

where $\boldsymbol{\nabla}_{\theta, \varphi} \hat{n}^{\alpha}=\partial \hat{n}^{\alpha} / \partial \theta \hat{\boldsymbol{\theta}}+(\sin \theta)^{-1} \partial \hat{n}^{\alpha} / \partial \varphi \hat{\boldsymbol{\varphi}}$ is perpendicular to $\hat{\boldsymbol{r}}$. This representation is equivalent to an expansion of $\boldsymbol{v}$ in terms of vector spherical harmonics $\boldsymbol{Y}$ and $\psi$.

This relation implies that the radial velocity $u(\boldsymbol{r})=\boldsymbol{v} \cdot \hat{\boldsymbol{r}}$ and $\mathrm{d} \phi / \mathrm{d} r$ are related by

$$
\frac{\mathrm{d} \phi^{\alpha}}{\mathrm{d} r}=-\frac{3}{4 \pi} \int u(\boldsymbol{r}) \hat{n}^{\alpha} \mathrm{d} \Omega .
$$

As an example for the representation in terms $\hat{n}^{\alpha}$, con- 
sider a constant velocity field $\boldsymbol{v}=B_{0} \hat{\boldsymbol{z}}$ in the z-direction. In this case, $\phi=\phi^{z} \hat{n}^{z}$ where $\phi^{z}(r)=-B_{0} r$. A substitution of this potential in 4 gives $\boldsymbol{v}=B_{0} \cos \theta \hat{\boldsymbol{r}}_{-} B_{0} \sin \theta \hat{\boldsymbol{\theta}}$ which gives $B^{x}=B^{y}=0$ and $B^{z}=B_{0}$, as expected.

Both terms on the r.h.s in the relation (4) contribute to $\mathbf{B}$ in (1). We could integrate the relation over a sphere in order to get the bulk in terms of $\phi^{\alpha}$. However, a much more elegant way of achieving the same thing is via the divergence theorem, which gives (Nusser et al. 2014)

$$
\mathbf{B}(r)=-\frac{3}{4 \pi r^{3}} \int_{S} \phi(\boldsymbol{r}) \mathrm{d} \boldsymbol{S},
$$

where the integration is over the surface of the sphere of radius $r$, with surface element $\mathrm{d} \boldsymbol{S}=\mathrm{d} \Omega r^{2} \hat{\boldsymbol{r}}$. Substituting (2) for $\phi(\boldsymbol{r})$, this relation gives

$$
B^{\alpha}=-\frac{\phi^{\alpha}}{r} .
$$

where $B^{\alpha}$ correspond to the three Cartesian components $B_{x}, B_{y}$ and $B_{z}$. The relation (6) also gives the bulk flow of a thin spherical shell of radius $r$ as

$$
B_{\mathrm{sh}}^{\alpha}=-\left[\frac{\mathrm{d} \phi^{\alpha}}{\mathrm{d} r}+2 \frac{\phi^{\alpha}}{r}\right] .
$$

\section{BULK FLOWS FROM ML}

Assume we are provided with galaxy positions $\boldsymbol{r}_{i}$ and radial peculiar motions $u_{i}$ of $i=1 \cdots N$ galaxies. The $1 \sigma$ error on $u_{i}$ is $\sigma_{i}$ and we assume that the positions are given accurately. The latter assumption can be justified if we use the redshifts as proxy to $r_{i}$ rather than the observed distances. ML provides an estimate, $\tilde{\mathbf{B}}$, of the bulk flow by minimizing

$$
\chi^{2}=\sum_{i} \frac{w_{i}}{\sigma_{i}^{2}}\left[u_{i}-\tilde{\mathbf{B}} \cdot \hat{\boldsymbol{r}}_{i}\right]^{2},
$$

where we allow for a weighting the galaxies by $w_{i}$ in addition to the usual statistical weights dictated by $\sigma_{i}$. At the minimum, $\partial \chi^{2} / \partial \tilde{B}^{\alpha}=0$ yields

$$
\sum_{\beta} \sum_{i} w_{i} \sigma_{i}^{-2} \hat{n}_{i}^{\alpha} \hat{n}_{i}^{\beta} \tilde{B}^{\beta}=\sum_{i} w_{i} \sigma_{i}^{-2} u_{i} \hat{n}_{i}^{\alpha}
$$

where we have used $\tilde{\mathbf{B}} \cdot \hat{\boldsymbol{r}}_{i}=\sum_{\beta} \tilde{B}^{\beta} \hat{n}^{\beta}$. Observational errors typically depend on distance and not the angular position, hence $\sigma_{i}=\sigma\left(r_{i}\right)$. If further no angular selection is imposed on the observed galaxies and $w_{i}=w\left(r_{i}\right)$, then the continuous limit of $(10)$ is

$$
\tilde{B}^{\alpha}=\frac{3 \int \mathrm{d} r^{\prime} r^{\prime 2} \mathrm{~d} \Omega \frac{w\left(r^{\prime}\right) \mathcal{N}\left(r^{\prime}\right)}{\sigma^{2}\left(r^{\prime}\right)} u\left(\boldsymbol{r}^{\prime}\right) \hat{n}^{\alpha}\left(\boldsymbol{r}^{\prime}\right)}{4 \pi \int \mathrm{d} r^{\prime} r^{\prime 2} \mathrm{~d} \Omega \frac{w(r) \mathcal{N}\left(r^{\prime}\right)}{\sigma^{2}(r)}},
$$

where $\mathcal{N}(r)$, is the 3D number density of observed galaxies. We ignore here contribution of the underlying clustering of matter and thus the dependence of $\mathcal{N}$ on $r$ is entirely due to observational selection strategy.

\section{THE INCONSISTENCY AND ITS RESOLUTION}

According to (11), if $u(\boldsymbol{r})=\mathbf{B}_{0} \cdot \hat{\boldsymbol{r}}$ where $\mathbf{B}_{0}$ is constant throughout the sphere, then we recover $\tilde{\mathbf{B}}=\mathbf{B}_{0}$. However, the estimate in 11 does not generally agree with the definition of the bulk flow as given in 11 . To see this we rewrite (7) as $B^{\alpha}=-\phi^{\alpha} / r=-\left(\int \mathrm{d} r^{\prime} \mathrm{d} \phi^{\alpha} / \mathrm{d} r^{\prime}\right) / r$ and note the relation between $u$ and $\mathrm{d} \phi^{\alpha} / \mathrm{d} r$ in (5). Hence, for a general choice of $w$, the mathematical relation (7) and the ML estimate (11) are inconsistent. Nonetheless, the two equations become consistent for the specific choice

$$
w=\frac{\sigma^{2}}{\mathcal{N} r^{2}} .
$$

The term $\mathcal{N} r^{2}$ could be identified with the number density per unit radius. Since typically $\sigma \propto r$, the weighting is essentially equivalent to $w \sim 1 / \mathcal{N}$. Minimizing $\chi^{2}$ in (9) with the weights, $w$, given by 12 will yield $\tilde{\mathbf{B}}(r)$ that is consistent (up-to statistical error) with the definition of the bulk flow as the average peculiar velocity within the sphere. In the absence of errors, this choice of $w$ guarantees that $\tilde{\mathbf{B}}(r)$ coincides with the true $\mathbf{B}(r)$.

Another way to achieve an estimate which agrees with the definition (1) is as follows. Let us divide space into a finite number, $s=1 \cdots N_{s}$, of spherical shells each of radius $r_{s}$ and thickness, $\delta_{s} \ll r_{s}$. Define a new $\chi^{2}$ function

$$
\chi^{2} \equiv \sum_{s=1}^{N_{s}} \sum_{i \in s} \sigma_{i}^{-2}\left[u_{i}-\sum_{\alpha} V_{s}^{\alpha} \hat{n}^{\alpha}\right]^{2} .
$$

Here the symbol $i \in s$ implies galaxies lying inside the shell $s$ and $V_{s}^{\alpha}=V^{\alpha}\left(r_{s}\right)$, where $V^{\alpha}=-\mathrm{d} \phi^{\alpha} / \mathrm{d} r$. Minimization of this $\chi^{2}$ with respect to $V_{s}^{\alpha}$ gives

$$
\sum_{\beta} \sum_{i \in s} \sigma_{i}^{-2} \hat{n}_{i}^{\alpha} \hat{n}_{i}^{\beta} V_{s}^{\beta}=\sum_{i \in s} \sigma_{i}^{-2} u_{i} \hat{n}_{i}^{\alpha} .
$$

Once $V_{s}^{\alpha}$ are obtained by solving the last equations, the potential can be computed as $\phi_{s}^{\alpha}=-\sum \delta r_{s} V_{s}^{\alpha}$ and the bulk flow of a sphere of radius $r_{s}$ identified as $B^{\alpha}=-\phi_{s}^{\alpha} / r_{s}$. Note that $V_{s}^{\alpha}$ coincides with the bulk flow of the shell only if the velocity in the shell is constant, otherwise, it will be missing the term $-2 \phi^{\alpha} / r$ as is seen from (8).

\section{A NUMERICAL DEMONSTRATION}

We give a demonstration for the case of perfect data with zero errors and uniform spatial coverage. We do that with the help of a random gaussian realization of a velocity field with a power spectrum of the $\Lambda$ CDM model with density parameters $\Omega_{c}=0.225, \Omega_{b}=0.045$, and $\Omega_{v}=0.73$, respectively, for the dark matter, baryons and the cosmological constant. The field is generated on a $512^{3}$ uniform gris in a box of $500 h^{-1} \mathrm{Mpc}$ on the side, with $H_{0}=70 \mathrm{~km} \mathrm{~s}^{-1} \mathrm{Mpc}^{-1}$. A grid point with a velocity close to the observed motion of the Local Group is chosen as the central "observer". The $3 \mathrm{D}$ velocities at the grid points within a distance of $100 h^{-1} \mathrm{Mpc}$ from the observer are used to directly compute the true bulk flow, $\mathbf{B}_{\mathrm{t}}$, of spheres centered on the observer. The actual radial velocities at the grid points are used as "observational" data, without any dilution and and any added noise. Thus, $\mathcal{N}(r)=$ const and $\sigma_{i}$ is a constant which formally is taken as very close to zero. We then derive two estimates for bulk flows for spheres around the observer. The first estimate is derived using the standard $\mathrm{ML}$ as appropriate for this data (i.e. $w \mathcal{N} / \sigma^{2}=$ const 


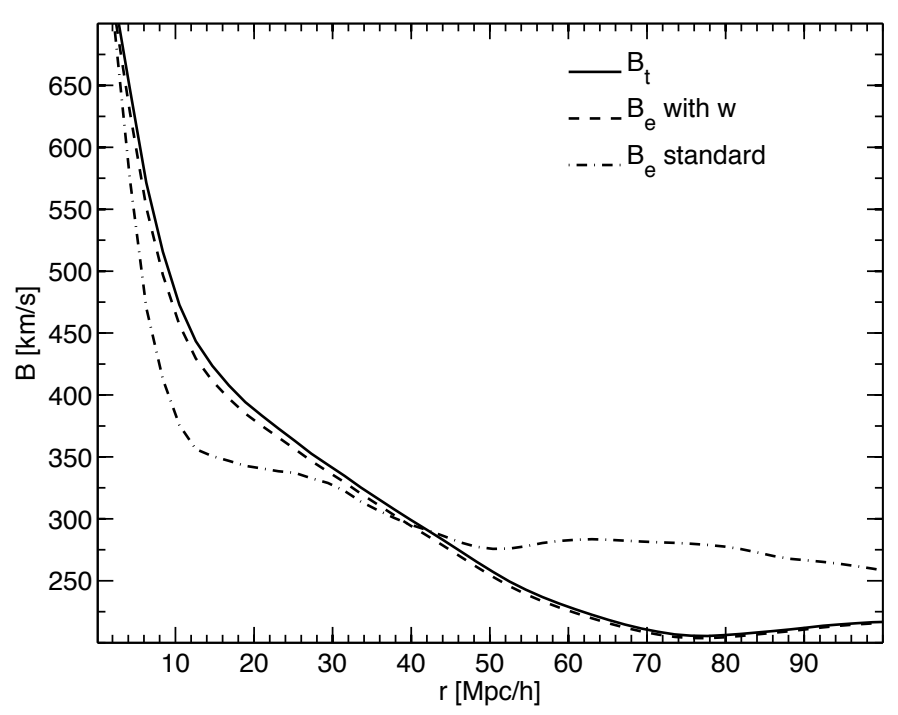

FIG. 1.- Estimation of the bulk flow for ideal data taken from a realization of a random gaussian velocity field. The bulk flow estimated with the weighting $w$ given by 12 agrees well with the true flow $\mathbf{B}_{t}$.

in eq. 11) and the second is obtained with the modified weighting in 12 (i.e. $w \mathcal{N} / \sigma^{2}=1 / r^{2}$ in eq. 11). The two estimates and the true bulk flow are shown in the Figure as a function of the radius. The discrepancy between the standard ML estimate and the true bulk flow is substantial, while the modified weighting almost yields perfect agreement. It is interesting that the two estimates would coincide had the data been diluted to $\mathcal{N} \propto r^{-2}$.

\section{GENERAL REMARKS}

The inconsistency in the ML estimation pointed out here stems from the assumption of a constant $\mathbf{B}$ in the survey volume. With this assumption, standard ML estimation yields, by definition, the most likely constant velocity vector which fits the data inside the survey volume. However, this constant velocity does not coincide with the definition of the bulk flow as the mean velocity of the relevant volume.

We do not aim here at quantifying the inconsistency for realistic data. Datasets are available with numerous version, each with its own peculiar characteristics and the differences between results of various weightings should be assessed individually. Further, any weighting scheme could be applied to a given dataset as long as the implications are assessed self-consistently within the context of a cosmological model or in comparison with other datasets. However, to avoid confusion the term "bulk flow" should be reserved to estimates of the mean motion rather than any other moment of the data.

Peculiar velocity data could be analyzed in many ways (e.g. Davis et al. 2011) which do not resort to an application of the ML estimation as presented above. The constrained realizations method (e.g Hoffman \& Ribak 1991: Yepes et al. 2014) reconstructs a full 3D velocity field from observed radial velocity data. In this method the bulk flow can be computed directly from the reconstructed 3D velocity field.

\section{ACKNOWLEDGMENT}

This research was supported by the I-CORE Program of the Planning and Budgeting Committee, THE ISRAEL SCIENCE FOUNDATION (grants No. 1829/12 and No. 654/13) and the Asher Space Research Institute. The author thanks Enzo Branchini and Martin Feix for comments which helped improving the manuscript.

\section{REFERENCES}

Davis, M., Nusser, A., Masters, K. L. Springob, C., Huchra J. P., \& Lemson, G. 2011, MNRAS, 413, 2906

Hoffman, Y., \& Ribak, E. 1991, ApJL, 380, L5

Kaiser, N. 1988, MNRAS, 231, 149

Lynden-Bell, D., Faber, S. M., Burstein, D., Davies, R. L. Dressler, A., Terlevich, R. J., \& Wegner, G. 1988, ApJ, 326, 19

Masters, K. L., Springob, C. M., Haynes, M. P., \& Giovanelli, R. 2006, ApJ, 653, 861

Nusser, A., Davis, M., \& Branchini, E. 2014, ArXiv:1402.6566
Tully, R. B., Courtois, H. M., Dolphin, A. E., Fisher, J. R., Héraudeau, P., Jacobs, B. A., Karachentsev, I. D., Makarov, D., Makarova, L., Mitronova, S., Rizzi, L., Shaya, E. J., Sorce, J. G., \& Wu, P.-F. 2013, AJ, 146, 86

Yepes, G., Gottloeber, S., \& Hoffman, Y. 2014, New Astronomy, 58,1 